\title{
Self-oscillating gels beating like a heart muscle
}

\author{
Ryo Yoshida ${ }^{1}$ \\ ${ }^{1}$ Department of Materials Engineering, School of Engineering, The University of Tokyo, 7-3-1 Hongo, Bunkyo-ku, Tokyo 113-8656, Japan
}

Received October 1, 2012; accepted October 18, 2012

\begin{abstract}
So far stimuli-responsive polymer gels and their application to smart materials have been widely studied. On the other hand, as a novel biomimetic gel, we developed gels with an autonomous self-oscillating function like a heart muscle, which was firstly reported in 1996. We designed the self-oscillating polymers and gels by utilizing the oscillating reaction, called the Belousov-Zhabotinsky (BZ) reaction. The self-oscillating polymer is composed of a poly( $\mathrm{N}$-isopropylacrylamide) network in which the catalyst for the $\mathrm{BZ}$ reaction is covalently immobilized. In the presence of the reactants, the polymer gel undergoes spontaneous cyclic swelling-deswelling changes without any on-off switching of external stimuli. Potential applications of the self-oscillating polymers and gels include several kinds of functional material systems, such as biomimetic actuators and mass transport surface. In this review, recent progress on the polymer gels is introduced.
\end{abstract}

Key words: Polymer gels, Self-oscillation, Biomimetics, Belousov-Zhabotinsky reaction

In a research field of polymer science, polymer gels have made rapid progress during the past 30 years, and they have played important roles in the field of biomaterials and biomimetics, etc. Since the discovery of "volume phase transition" phenomena ${ }^{1}$ as a turning point, many researchers have developed several kinds of stimuli-responsive polymer gels that exhibit reversible swelling-deswelling change in response to environmental changes such as solvent composition, temperature, $\mathrm{pH}$ change, etc. Especially, from the late 1980's or

Corresponding author: Ryo Yoshida, Department of Materials Engineering, School of Engineering, The University of Tokyo, 7-3-1 Hongo, Bunkyo-ku, Tokyo 113-8656, Japan.

e-mail: ryo@cross.t.u-tokyo.ac.jp early 1990's, new functional gels have been developed as "intelligent gels" or "smart gels", and their applications have been demonstrated such as actuator (artificial muscle), drug delivery systems (DDS), tissue engineering, purification or separation systems, biosensor, shape memory materials, molecular recognition systems, etc. ${ }^{2-5}$. A concept of "chemo-mechanical gels" to convert chemical energy of chemical or biochemical reaction to mechanical energy of gels has been proposed since a long time ago, as a model of artificial muscles to realize soft actuators. Recently, gels have been used in the field of micromachines and nanotechnology. In addition to new synthetic methods to give unique functions by molecular design in nano-order scale including supramolecular design, the design and construction of micro or nanomaterial systems with the biomimetic functions have been attempted.

One of the characteristic behaviors in living systems is autonomous oscillation, that is, spontaneous changes with temporal periodicity (called "temporal structure") such as heartbeat, brain waves, pulsatile secretion of hormone, cell cycle, and biorhythm. Although several stimuli-responsive polymer systems have been studied inspired by living systems, there are few studies on polymer systems undergoing self-oscillation under constant condition without any on-off switching of external stimuli. If such autonomous polymer systems can be realized by using completely synthetic polymers, unprecedented biomimetic materials may be created.

\section{Design of self-oscillating polymer gels}

From this viewpoint, we have studied the polymer gels with autonomous function. In contrast to conventional stimuliresponsive gels, we developed a novel "self-oscillating" polymer gel that exhibits autonomous mechanical oscillation without an external control in a completely closed solution (Fig. 1). For the design of the gel, the Belousov- 
Swelling

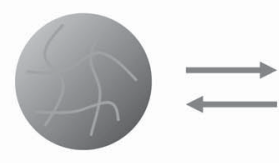

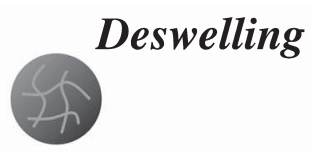

"Stimuli-responsive" gel

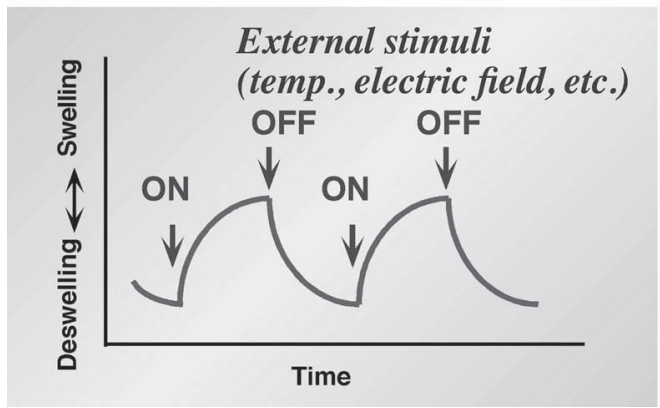

"Self-oscillating" gel

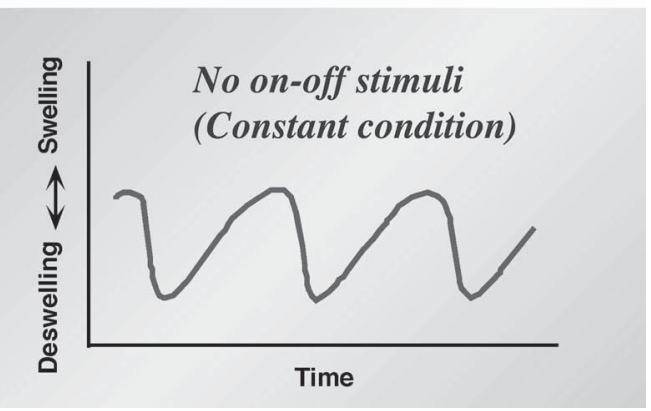

Figure 1 Stimuli-responsive and self-oscillating gels.

Zhabotinsky (BZ) reaction ${ }^{6-8}$, which is well-known for exhibiting temporal and spatiotemporal oscillating phenomena, was focused. The overall process is the oxidation of an organic substrate, such as malonic acid (MA) or citric acid, by an oxidizing agent (bromate ion) in the presence of a strong acid and a metal catalyst. In the course of the reaction, however, the catalyst undergoes spontaneous redox oscillation. When the solution is homogeneously stirred, the color of the solution periodically changes, based on the redox changes of the metal catalyst. When the solution is placed as a thin film in stationary conditions, concentric or spiral wave patterns develop in the solution. The wave of oxidized state propagating in the medium at a constant speed is called a "chemical wave". The reaction is often analogically compared with the TCA cycle which is a key metabolic process taking place in the living body, and it is recognized as a chemical model for understanding several autonomous phenomena in biological systems.

We attempted to convert the chemical oscillation of the $\mathrm{BZ}$ reaction into a mechanical change in gels and generate an autonomous swelling-deswelling oscillation under nonoscillatory outer conditions. A copolymer gel consisting of $\mathrm{N}$-isopropylacrylamide (NIPAAm) and ruthenium tris(2,2'bipyridine) $\left(\mathrm{Ru}(\mathrm{bpy})_{3}\right)$, acting as a cataly for the $\mathrm{BZ}$ reaction, was prepared (Fig. 2(a)). When the poly(NIPAAm-co$\mathrm{Ru}(\mathrm{bpy})_{3}$ ) gel is immersed in the catalyst-free BZ solution, the reaction occurs in the gel by the catalytic function of the polymerized $\mathrm{Ru}(\mathrm{bpy})_{3}$ (Fig. 2(c)). The redox changes of the polymerized catalyst moiety (Ru(bpy) ${ }_{3}{ }^{2+} \leftrightarrow \mathrm{Ru}(\mathrm{bpy})_{3}{ }^{3+}$ ) change the volume phase transition temperature as well as the swelling ratio of the gel because the hydrophilicity of the polymer chains increases at the oxidized $\mathrm{Ru}(\mathrm{III})$ state and decreases at the reduced Ru(II) state (Fig. 2(b)). As a result, the gel exhibits an autonomous swelling-deswelling oscillation with the redox oscillation in the closed solution under constant condition (Fig. 2(d)). Since being first reported in 1996 as a "self-oscillating gel", we have been systematically studying the self-oscillating polymer and gel as well as their applications to biomimetic or smart materials ${ }^{10-34}$.

\section{Self-oscillating behaviors of the polymers and the gels}

As shown in Figure 2(d), self-oscillation can be induced on several scales. In the case of the uncrosslinked linear polymer, the polymer solution exhibits self-oscillations of optical transmittance and viscosity due to spontaneous cyclic soluble-insoluble changes of the polymer. When the gel is enough small, redox changes occur homogeneously in the gel without pattern formation ${ }^{11}$. Then the swelling-deswelling change of the gel becomes isotropic. On the other hand, when the gel size is much larger than the wavelength of the chemical wave, a train of excited pulses of the oxidized state (i.e. "chemical waves") spontaneously evolves and propagates in the gel by a reaction-diffusion mechanism. In the case of a 2-dimensional gel sheet, concentric or spiral waves can be observed. With the propagation of chemical waves, the self-oscillating gel exhibits peristaltic motion ${ }^{12,13}$, that is, the locally swollen (or shrunken) region corresponding to a locally oxidized (or reduced) state propagates in the gel, similar to the locomotion of a living worm.

The period and amplitude of the gel or polymer oscillation can be controlled by changing substrate concentration and temperature. Further, on-off regulation of the selfoscillation by external stimuli such as temperature change, addition and removal of organic acid, photo-irradiation is possible $^{10}$. In particular, $\mathrm{Ru}(\mathrm{bpy})_{3}$ has photo-sensitivity at the reduced state. By photo-irradiation, it creates the other reaction pathway to stop or cause the oscillation by producing an intermediate to act as an inhibitor $\left(\mathrm{Br}^{-}\right)$or an acti- 


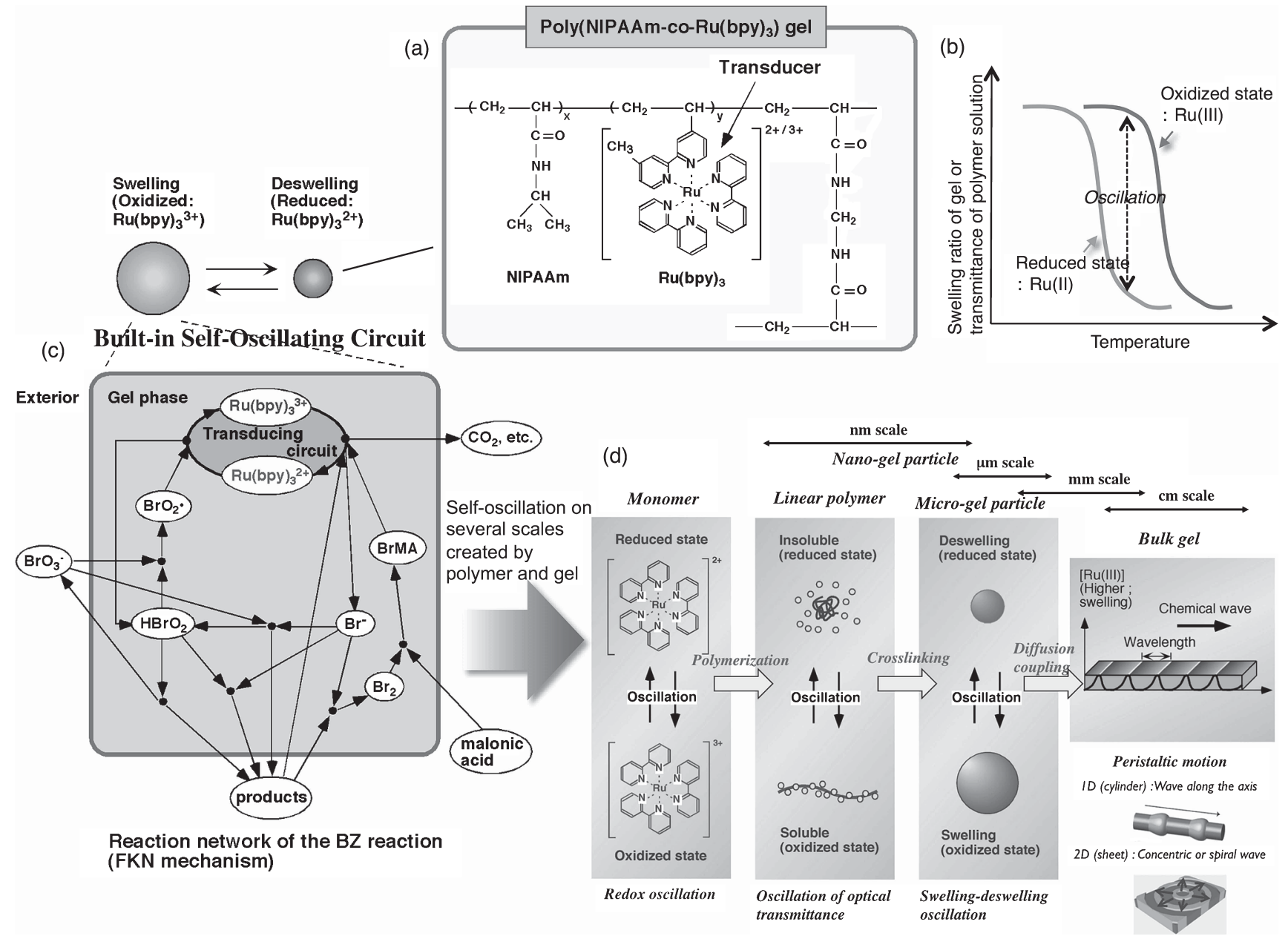

Figure 2 Mechanism of self-oscillation and the self-oscillating behavior on several scales.

vator $\left(\mathrm{HBrO}_{2}\right)$, depending on the composition of the $\mathrm{BZ}$ solution $^{8}$. By utilizing such photochemical characteristics of the BZ reaction, we experimentally demonstrated photoregulation of self-oscillating motion of the gel ${ }^{13,14}$. Further, Balazs et al. ${ }^{35,36}$ demonstrated light-guided motility of the gel by theoretical model simulation.

Recently, we designed new photo-regulated self-oscillating systems based on hydration and dehydration change of the polymer by photo-irradiation. Spirobenzopyran was introduced into the self-oscillating polymer as the photochromic site. Under acidic condition necessary for the BZ reaction, photochromism of the spirobenzopyran occurs and the lower critical solution temperature (LCST) of the polymer solution shifts to lower temperature with isomerization by photo-irradiation. As a result, on-off switching of the soluble-insoluble self-oscillation for the polymer solution is possible by photo-irradiation. This result suggests that spatio-temporal photo-regulation of self-oscillating mechanical motion is possible for the gel.

\section{Design of biomimetic soft-actuators}

Such macroscopic swelling-deswelling oscillation may lead to the creation of novel biomimetic actuators. As applications to autonomous biomimetic actuators, ciliary motion actuators (artificial cilia) ${ }^{15}$ and self-walking gels ${ }^{16}$, etc. were realized (Fig. 3). Further, self-propelled motion of gels was theoretically demonstrated and experimentally realized by utilizing the gels ${ }^{17}$. The cylindrical self-oscillating gel with a gradient in crosslinking density was prepared by photopolymerization. To introduce gradient structure, during the gelation, light was irradiated from one side to the pre-gel solution in a grass capillary. The resulting gel has denser crosslinking at the photo-irradiated side, and bends to the direction at the reduced state. In the catalyst-free BZ solution, the gel repeated bending and stretching motion, and the position of the center of the gel apparently shifts slowly upwards (towards the region with lower cross-link density). In addition, we attempted to control the motion by preparing a composite gel of self-oscillating and non-active gels ${ }^{18}$. For this purpose, heterogeneous BZ gel was prepared. The disk- 


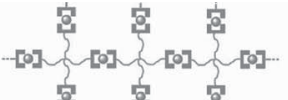

$$
\begin{aligned}
& \mathrm{Ru}(\mathrm{III}) \downarrow \uparrow \mathrm{Ru}(\mathrm{II}) \\
& \text {-[0] }
\end{aligned}
$$

Self-oscillation of complex formation
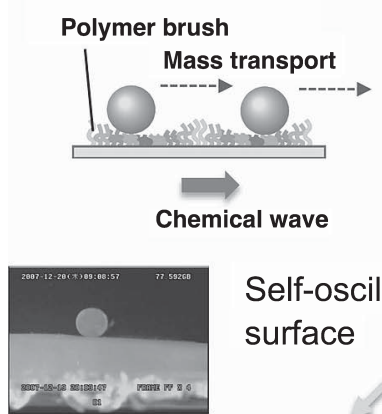

Self-oscillating surface

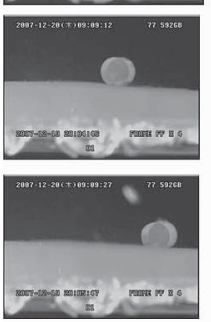

Mass transport surface

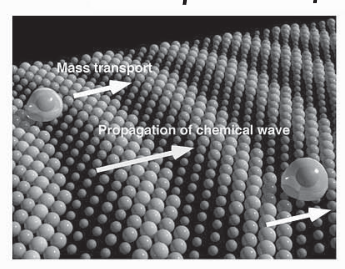

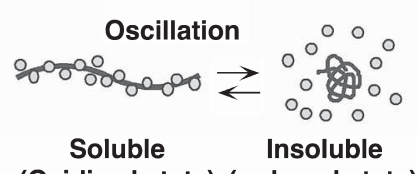

(Oxidized state) (reduced state)

Soluble-insoluble oscillation of polymer chains

Functional fluid

- Transmittance oscillation

-Viscosity oscillation

\section{Self-oscillating gel}
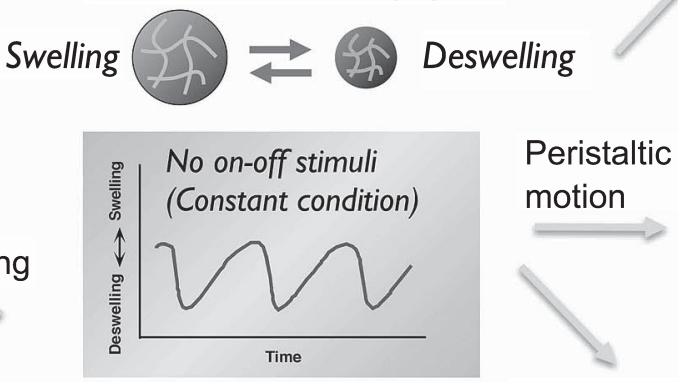

\section{Biomimetic actuator}

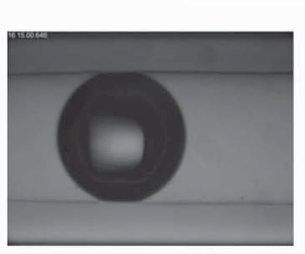

Transport tube

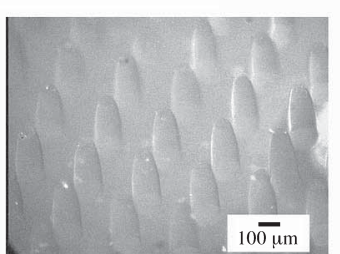

Artificial cilia

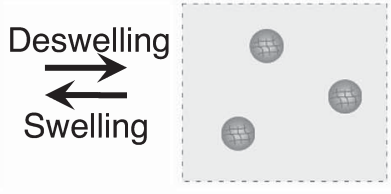

Swollen state (Oxidized) Shrunken state (Reduced) Dispersed Dispersed
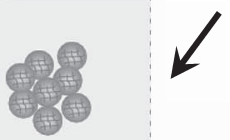

Shrunken state (Reduced) Flocculated

Figure 3 Development of self-oscillating polymer gels.

shaped self-oscillating gels were arranged around the corners of a polygonal sheet of poly(acrylamide) (PAAm) gel. Wave propagation was controlled by changing the patch size, catalyst content of the BZ gel, and spacing between the patches. Balazs et al. ${ }^{37,38}$ developed a mathematical model for simulating chemomechanical behaviors of the selfoscillating gels. They have demonstrated several aspects of the self-oscillating behavior for the gel by theoretical simulation. Many interesting phenomena are demonstrated theoretically. The findings from these studies provide guidelines for creating autonomously moving objects, which can be used for robotic or microfluidic applications.

\section{Soft actuators composed of assembled self- oscillating microgels}

In a living muscle, there exists hierarchical structure to amplify the microscopic movements of actin-myosin to macroscopic displacements. Suzuki et al. ${ }^{19}$ introduced such a hierarchical structure into the self-oscillating gel to get large amplitude of swelling/deswelling oscillation. The soft actuators assembled from self-oscillating submicron-sized hydrogel particles (microgels) were designed and obtained by assembling the pre-existing microgel particles. The amplitude of the actuator's oscillation was controlled by selecting the types of oscillations of constituent microelements, i.e., swelling/deswelling or cooperative dispersing/flocculating motion of the microgels. They exhibited large displacements due to cooperative dispersing/flocculating motion of constituent microelements. The new actuators prepared by this simple fabrication process make the oscillating actuators suitable for practical use in microfluidic devices such as self-beating micropumps.

\section{Design of autonomous mass transport systems}

\subsection{Self-driven gel conveyer:}

Autonomous transportation on the self-oscillating gel surface by peristaltic motion

Further, in order to realize self-driven gel conveyer as novel autonomous mass transport system, we attempted to transport an object by utilizing the peristaltic motion of the 

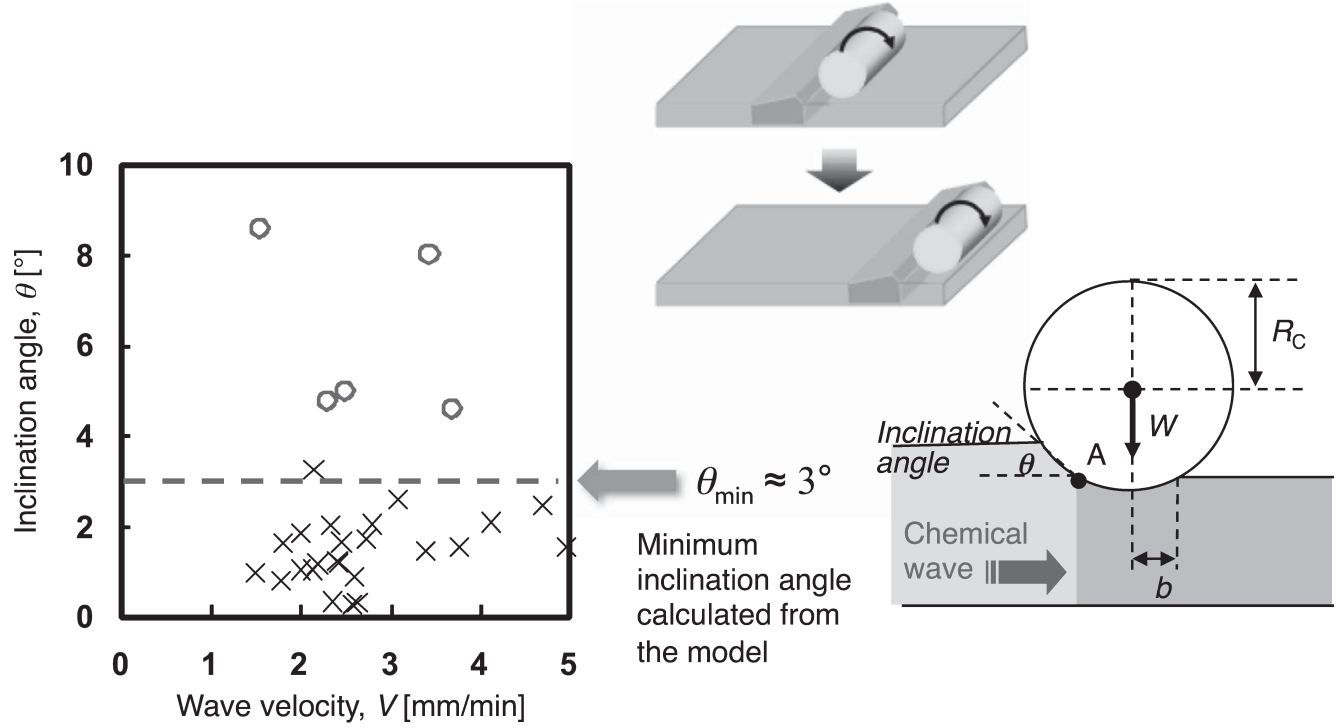
calculated from the model

Figure 4 (Left) Phase diagram of the transportable region given by the velocity and the inclination angle of the wave front: $(\mathrm{O})$ transported, $(\times)$ not transported. (Right) Model of the rolling cylindrical gel on the peristaltic gel surface $\left(\mathrm{R}_{\mathrm{C}}=\right.$ radius of curvature, $\mathrm{W}=$ load of the PAAm gel, $\mathrm{b}=$ contact half-width).

self-oscillating gel (Fig. 3). It was found to be effective to copolymerize 2-acrylamido-2'-methylpropanesulfonic acid (AMPS) to a poly(NIPAAm-co-Ru(bpy) $)_{3}$ ) gel network to generate a large amplitude of volume change of the ge ${ }^{20}$. As a model object, a cylindrical poly(acrylamide) (PAAm) gel was put on the surface of self-oscillating gel sheet. It was observed that the PAAm gel was transported on the gel surface with the propagation of the chemical wave as it rolled (see the photograph in Fig. 3). Figure 4 (left) shows a phase diagram of transportable conditions given by the wave velocity and the inclination angle $\theta$ of the wavefront. The velocity and the inclination angle of the chemical wave were changed by changing the concentrations of the outer solution. For the controlled chemical waves with several inclination angles and velocities, whether the cylindrical gel could be transported or not was estimated. It was found that the cylindrical PAAm gel was not transported when the inclination angle was less than approximately $3^{\circ}$. The mass transportability did not depend on the velocity of the chemical wave, but on the diameter of the cylindrical PAAm gel and the inclination angle of the wave front.

For analyzing this result, we have proposed a model to describe the mass transport phenomena based on the Hertz contact theory, and the relation between the transportability and the peristaltic motion was investigated ${ }^{20,21}$. Figure 4 (right) shows the contact model of the cylindrical PAAm gel and the self-oscillating gel sheet. Here it is assumed that they are a non-adhesive rigid cylinder and an elastic plane, respectively, and there is no physical interaction between them. If the inclination angle was smaller than the slope of the tangent at point $\mathrm{A}$, sufficient contact force from the strain doesn't act to rotate the cylindrical PAAm gel. Therefore, the condition for the transport of the cylindrical PAAm gel is written as $R_{\mathrm{C}} \sin \theta \geq b$. The contact half-width, $b$, can be calculated from the Hertz contact theory by using the actual values of physical property for the loaded gel and the gel sheet such as Poisson ratio, the Young modulus, etc. As a result of calculation from the above equation, the minimum inclination angle was $3^{\circ}$ and it was the same as the angle resulted from the experiment. It was found experimentally and supported by the model that the sheer wave front of the peristaltic motion was necessary to transport cylindrical gels.

Further, to transport gel particles other than cylindrical gels, the self-oscillating gel sheet having a grooved surface was fabricated by using PDMS template. Poly(AAm-coAMPS) gel beads with the diameter of several hundred $\mu \mathrm{m}$ to several $\mathrm{mm}$ were transported on the grooved surface ${ }^{21}$. And also, instead of the homopolymer gel of PAAm, several kinds of copolymer gels consisting of AAm with 2acrylamido-2'-methylpropanesulfonic acid (AMPS), $N$-(3aminopropyl)methacrylamide hydrochloride (APMA), $\mathrm{N}$ (hydroxymethyl)acrylamide (HMAAm), and methyl methacrylate (MMA) were prepared as model cargos with different surface properties; positive or negative charge, more hydrophilicity or hydrophobicity, respectively. The influences of these surface properties on the transport behaviors were investigated $^{22}$. It was suggested that the adhesion force between the cylindrical poly(AAm-co-MMA) gel and the self-oscillating gel sheet was too strong to apply the transport model because of their hydrophobic interaction. The adhesive force to prevent transportation is not significant for the other gels, which agrees with the prediction from swelling, zeta potential, and contact angle measurements. Then we investigated the effect of surface roughness on the transportation. It was found that higher surface roughness is 


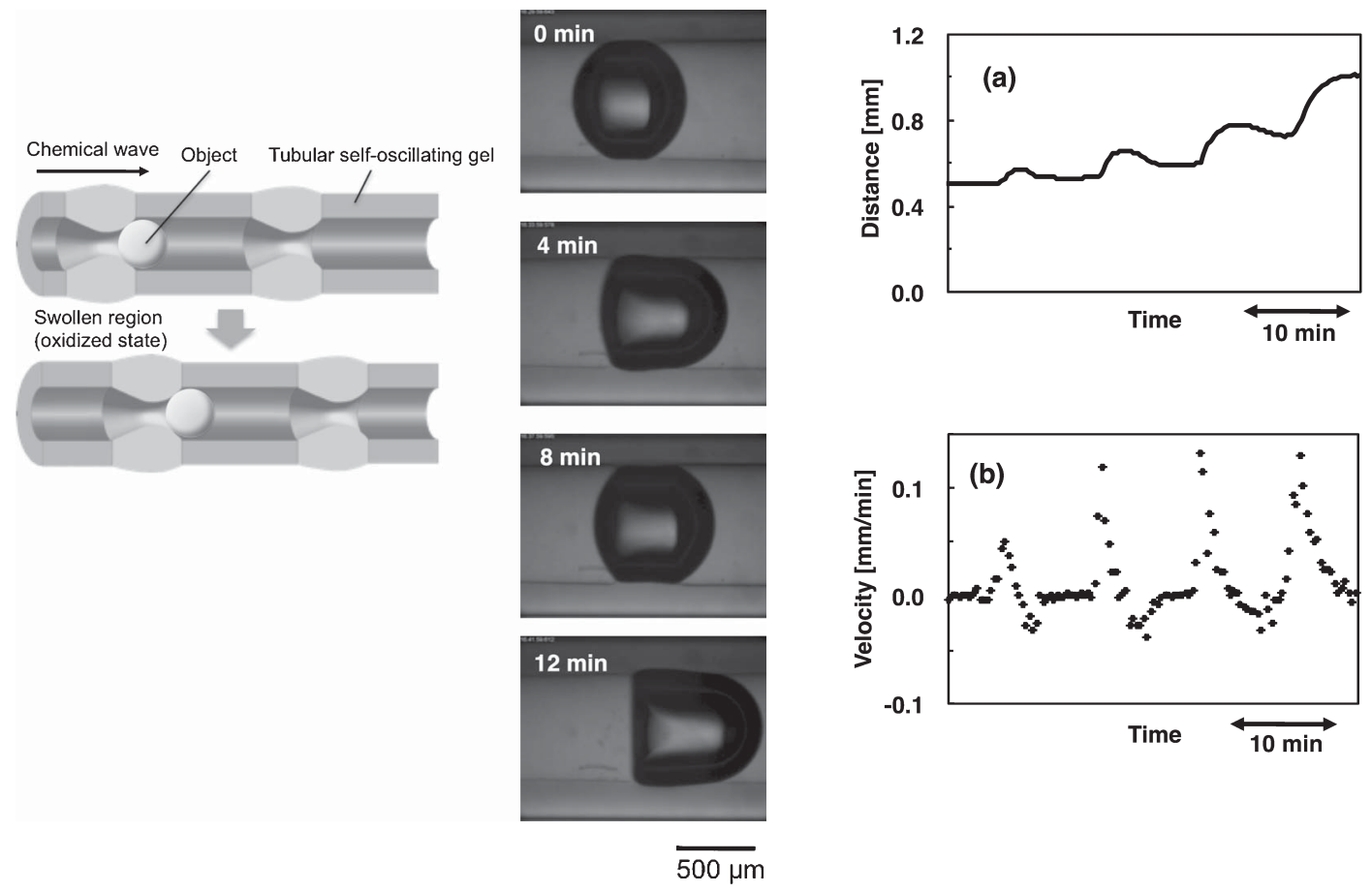

Figure 5 (Left) Schematic illustration of autonomous mass transport by peristaltic pumping of a tubular self-oscillating gel. (Center) The behavior of the autonomous transport of a $\mathrm{CO}_{2}$ bubble in the gel tube by peristaltic pumping. (Right) (a) Change in the position of the bubble. (b) The velocity of the bubble.

more effective in transporting the loaded gel because frictional force increases and the moment of force of the rotational motion increases. It is also possible to create a new dynamic interface by immobilizing the self-oscillating polymers. We prepared a self-oscillating polymer brush surface on the inside surface of a glass capillary and evaluated its dynamic behavior. Details will be shown in near future.

\subsection{Autonomous intestine-like motion of tubular self- oscillating gel}

Further, to construct autonomous mechanical pumping systems like an intestine, we fabricated the self-oscillating gel in a tubular shape ${ }^{24}$ (Fig. 5). Tubular self-oscillating gels were fabricated by photopolymerization. Different types of peristaltic motion were demonstrated by preparing a tubular gel that adheres to an inner wall of a glass capillary, a tubular gel that can swell freely without mechanical restraint, and a tubular IPN gel consisting of self-oscillating and non-oscillating polymer networks. In the gel tube, it was observed that a gas bubble was autonomously transported. Mass transport by peristaltic pumping of the tubular selfoscillating gel was successfully demonstrated.

Figure 5 (center) shows the behavior of a $\mathrm{CO}_{2}$ bubble in the tubular poly(NIPAAm-co-Ru(bpy) $)_{3}$ ) gel. The bubble moved intermittently by repeated deformation and restoration in the direction of chemical wave propagation. When the chemical wave reaches the contact point, the bubble is squashed and deformed by swelling of the gel layer at the point. Then the bubble is mechanically pushed forward by the peristaltic pumping mechanism. After the wave passes through, the gel layer deswells and the squashed bubble returns back to the initial round shape. Due to a decrease in pushing force and a negative pressure, the bubble moves backward slightly. After that, the movement of the bubble stops for a while. As a result, the movement was intermittent (Fig. 5 (right)). By repeating this process, the bubble is transported in the gel tube.

Recently, controlled transport of small objects has become one of the hot topics in many areas of science and engineering including chemistry, physics, biology, etc. Small objects such as particles with self-propelled motion are being actively studied, which may be the beginning of a new research trend. The autonomous transport by chemomechanical locomotion of the gel reported here is based on a different principle from the systems which have been reported so far. Potential applications to artificial intestines, artificial digestive tracts, etc. can be expected. Furthermore, there is a possibility of autonomous flow of an inner fluid. We are also investigating an application to a novel micropump for microfluidic systems.

\section{Self-oscillating polymer solution and microgel dispersion as functional fluids}

In the case of the uncrosslinked linear polymer, as shown in Figure 2(d), the polymer undergoes spontaneous cyclic 


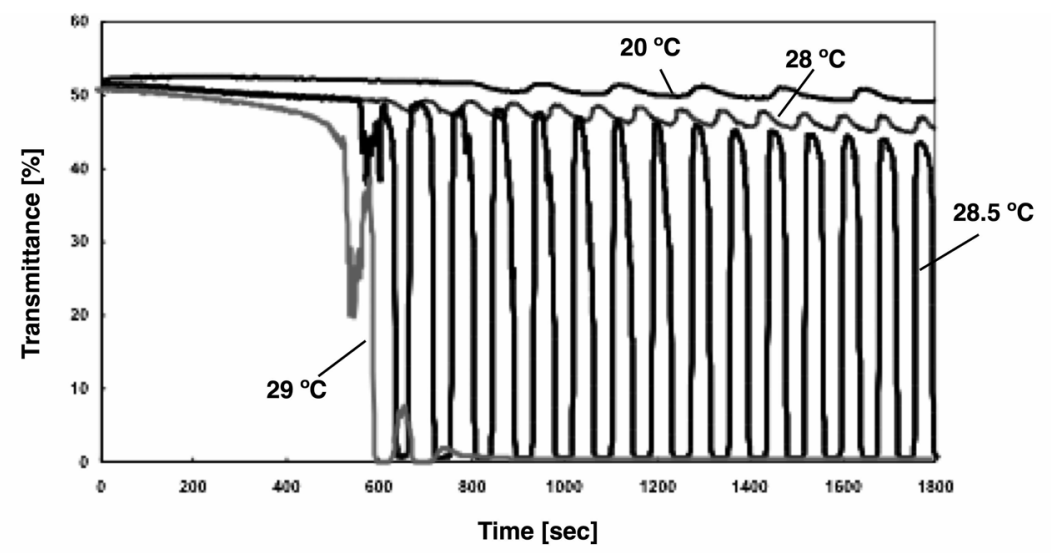

(a)

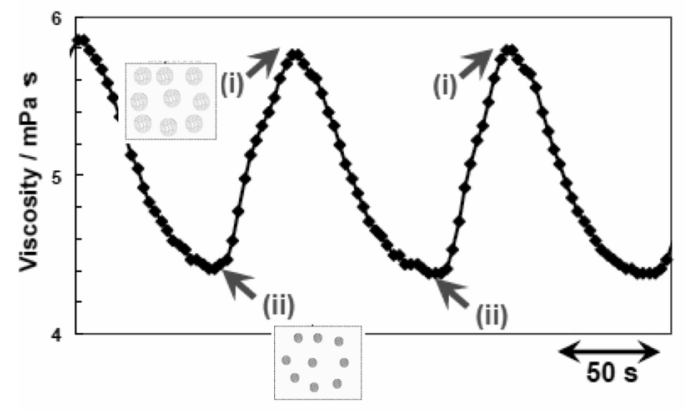

(b)

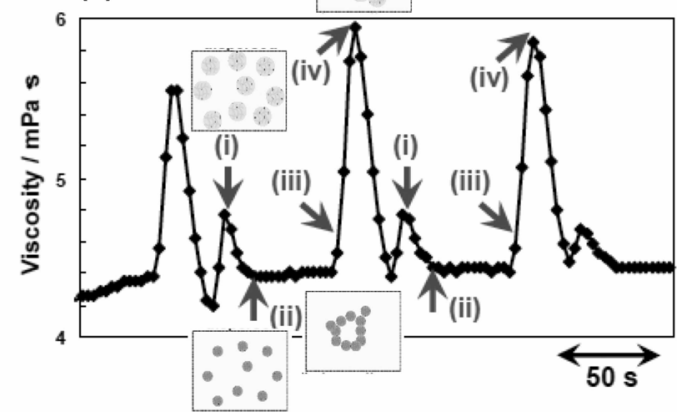

Figure 6 (Upper) Self-oscillating profiles of optical transmittance for the microgel dispersions at different temperatures. (Lower) Two different waveforms of viscosity oscillation of microgel dispersion observed at (a) $20^{\circ} \mathrm{C}$ and (b) $23^{\circ} \mathrm{C}$. The numbers in the figures denote (i) swollen and dispersed state, (ii) deswollen and dispersed state in Figure 6 (a), and (i) swollen and dispersed state, (ii) deswollen and dispersed state, (iii) deswollen and flocculated state, and (iv) swollen and flocculated state, in Figure 6 (b).

soluble-insoluble changes and the transmittance of the polymer solution oscillates autonomously ${ }^{25,26}$. In addition to analyzing the polymer solutions, we prepared submicron-sized poly(NIPAAm-co-Ru(bpy) $)_{3}$ ) gel beads by surfactant-free aqueous precipitation polymerization, and analyzed the oscillating behavior of the microgel dispersions ${ }^{27,28}$ (Fig. 6 (upper)). At low temperatures $\left(20-28^{\circ} \mathrm{C}\right)$, on raising the temperature, the amplitude of the oscillation became larger. The increase in amplitude is due to the increased deviation of the hydrodynamic diameter between the $\mathrm{Ru}(\mathrm{II})$ and $\mathrm{Ru}(\mathrm{III})$ states. Furthermore, a remarkable change in the waveform was observed between $28^{\circ} \mathrm{C}$ and $28.5^{\circ} \mathrm{C}$. Then, the amplitude of the oscillations dramatically decreased and finally the periodic transmittance changes could no longer be observed at $29^{\circ} \mathrm{C}$. The sudden change in oscillation waveform should be related to the difference in colloidal stability between the $\mathrm{Ru}(\mathrm{II})$ and $\mathrm{Ru}(\mathrm{III})$ states. Here, the microgels should flocculate due to the lack of electrostatic repulsion when the microgels were deswollen (see Fig. 1). The remarkable change in the waveform was only observed at higher dispersion concentrations (greater than $0.225 \mathrm{wt} . \%$ ). This self-oscillating property makes microgels more attractive for future developments such as microgel assembly, optical and rheological applications, etc. As mentioned before, the macroscopic self-oscillating gel prepared by crosslinking the assembled microgels exhibits a faster response and leads to larger amplitude of swelling-deswelling oscillation $^{19}$. And recently, self-oscillating core/shell microgels composed of the self-oscillating microgel as a core and PNIPAAm or poly ( $N$-isopropylmethacrylamide) (PNIPMAm) as a shell were proposed ${ }^{29}$. Using these microgels, the effects of crosslinked nanoshells covering self-oscillating microgels on their oscillating behaviors were investigated.

In the self-oscillating polymer solution or the microgel dispersion, viscosity oscillation as well as optical transmittance oscillation can be observed ${ }^{30-32}$. In the microgel dispersion, we found out that viscosity oscillation occurs in two different manners, exhibiting a simple pulsatile waveform or a complex waveform with two peaks per period (Fig. 6 (lower)). It was suggested that the difference in waveform is due to the difference in the oscillating manner of the microgels: swelling/deswelling or dispersing/flocculating oscillation as mentioned before. We can control the rhythm and amplitude of the oscillation by using these two phenomena of the microgels. and by changing the concentration of microgels, Ru(bpy) ${ }_{3}$ contents, crosslinking density, etc. It is expected that these polymer solutions and microgel dispersions are applied as novel functional fluid. 


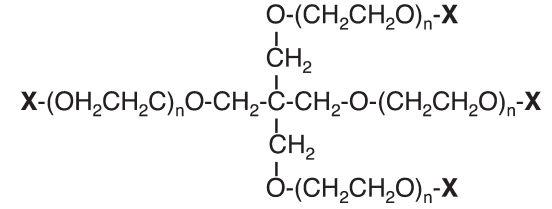

Terpyridine-terminated TetraPEG

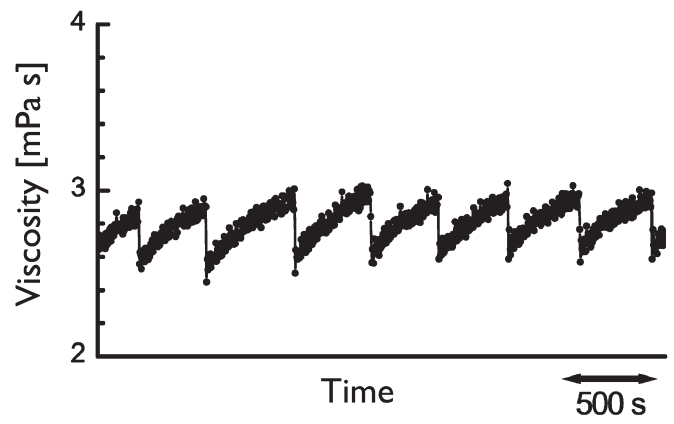

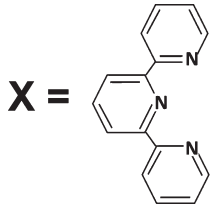

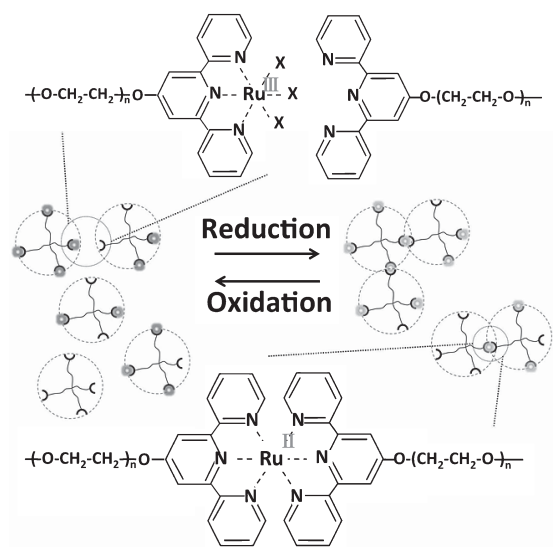

Figure 7 Autonomous viscosity oscillation by reversible complex formation of terpyridine-terminated tetra PEG in the BZ reaction. Oscillating profiles of viscosity of the aqueous solution containing $\mathrm{Ru}(\text { terpy })_{2}$-tetra $\mathrm{PEG}, \mathrm{HNO}_{3}, \mathrm{NaBrO}_{3}$ and $\mathrm{MA}$ at $25^{\circ} \mathrm{C}$.

\section{Autonomous viscosity oscillation by reversible complex formation of terpyridine-terminated $P E G$ in the $B Z$ reaction}

We also realized viscosity oscillations of a polymer solution based on different mechanisms ${ }^{33}$. It is known that a terpyridine ligand binds or dissociates a $\mathrm{Ru}$ metal ion depending on the redox states of the Ru metal ion. Generally, when the $\mathrm{Ru}$ metal ion is in the reduced $\mathrm{Ru}(\mathrm{II})$ state, the $\mathrm{Ru}(\mathrm{II})$ metal ion forms bis-complexes with terpyridine $\left(\mathrm{Ru}(\text { terpy })_{2}\right)$. However, when the Ru metal ion is in the oxidized $\mathrm{Ru}(\mathrm{III})$ state, the $\mathrm{Ru}(\mathrm{III})$ metal ion forms monocomplex with terpyridine ( $\mathrm{Ru}($ terpy)). Therefore, supramolecular block copolymers have been made by using $\mathrm{Ru}(\text { terpy })_{2}$ as a junction point ${ }^{39}$. If the Ru-terpyridine complex acts as a catalyst of the $\mathrm{BZ}$ reaction, the redox oscillation may cause periodical binding/dissociation of the Ru-terpyridine complex. Recently, a theoretical computational simulation in the case that the Ru-terpyridine complex acts as a reversible cross-linking point of polymer network during the BZ reaction has been reported by Balazs et $a .^{40}$. The swellingdeswelling oscillating behaviors of the gel were theoretically demonstrated by the simulation. When the crosslinking density is not high enough to form a gel, it is expected that the reversible complex formation causes a viscosity oscillation of the polymer solution due to a change in molecular weight.

We achieved autonomous viscosity oscillation by reversible complex formation of terpyridine-terminated PEG and/or terpyridine-terminated Tetra PEG in the $\mathrm{BZ}$ reaction ${ }^{33}$. Then the $\mathrm{BZ}$ reaction induces the periodical binding/dissociation of the Ru-terpyridine complex and causes periodic molecular changes and results in viscosity changes (Fig. 7). Differently from the viscosity oscillation we reported before, this mechanism based on complex formation may be advantageous in terms of the remarkable change in molecular weight. Although the amplitude of the viscosity oscillation in this experiment was still small for practical applications, we believe that remarkable changes like a sol-gel transition could be possible by controlling molecular design. We are investigating molecular design of terpyridine-terminated PEG for this purpose.

\section{Other attempts of self-oscillation toward applications}

So far, we have succeeded in developing a novel selfoscillating polymer (or gel) by utilizing the BZ reaction. However, the operating conditions for the self-oscillation are limited to conditions under which the BZ reaction occurs. For potential applications as functional bio- or biomimetic materials, it is necessary to design a self-oscillating polymer which acts under biological environments. To induce self-oscillation of polymer systems under physiological conditions, BZ substrates other than organic ones, such as malonic acid and citric acid, must be built into the polymer system itself. For this purpose, we have synthesized a quaternary copolymer which includes both $\mathrm{pH}$-control and oxidant-supplying sites in the poly(NIPAAm-co-Ru(bpy) $)_{3}$ ) chain at the same time ${ }^{26}$. By using this polymer, selfoscillation by adding only the organic acid (malonic acid) was actually observed. 

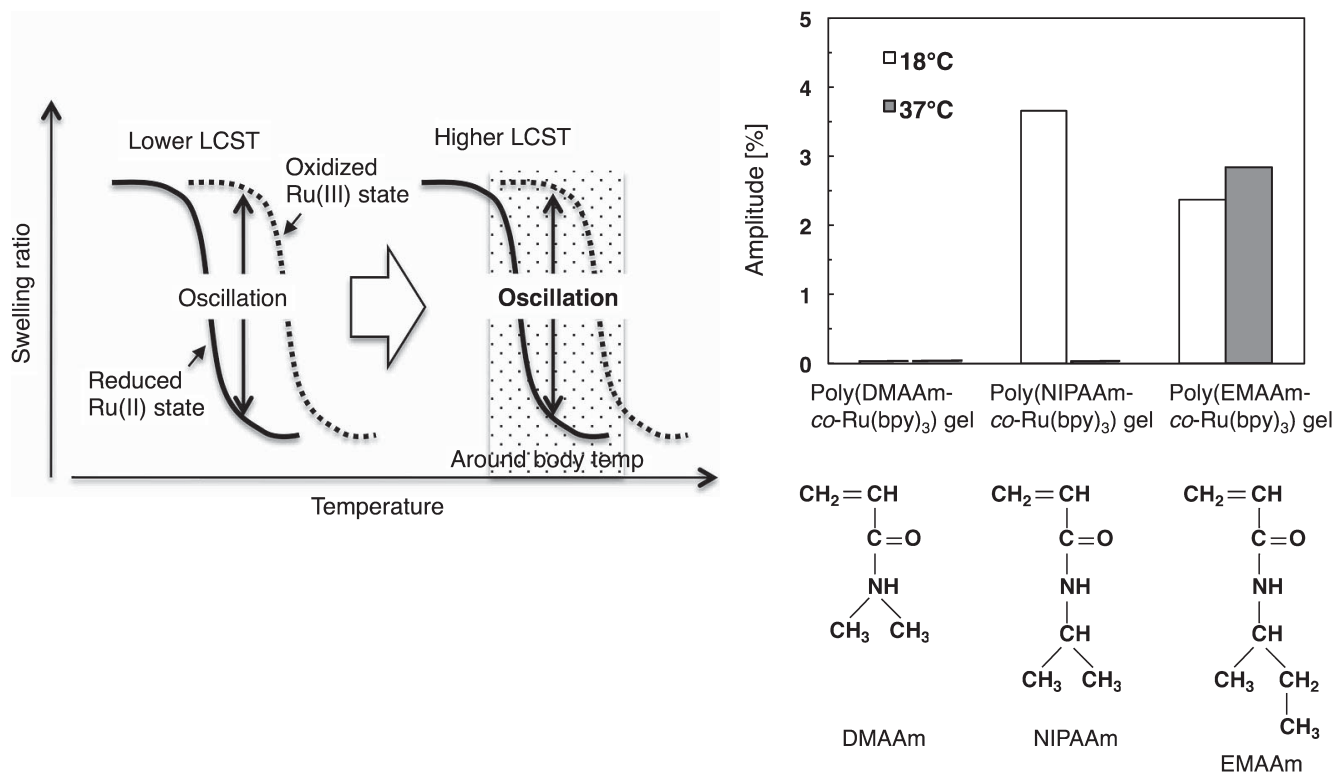

Figure 8 (Left) Strategy for achievement of self-oscillation at higher temperature while maintaining a large amplitude by utilizing a polymer with higher LCST. (Right) Comparison of oscillation amplitude of swelling-deswelling at $18^{\circ} \mathrm{C}$ and $37^{\circ} \mathrm{C}$ among the poly(NIPAAm-co-Ru(bpy) $\left.)_{3}\right)$ gel, the poly(EMAAm-co-Ru(bpy) $)_{3}$ ) gel and the poly(DMAAm-co-Ru(bpy $)_{3}$ ) gel.

Furthermore, it is desirable that the self-oscillation can be induced around body temperature. Typically the volume phase transition temperature of the poly(NIPAAm-co-Ru(bpy) $\left.)_{3}\right)$ gel is around $25^{\circ} \mathrm{C}$, and above that temperature the gel shrinks for both the reduced and oxidized states. As a result, it is difficult to induce self-oscillation near body temperature. For self-oscillation at higher temperatures, it is necessary to avoid the collapse of the polymer at those temperatures. One possible method may be to utilize a nonthermosensitive polymer without an LCST. In this case, the difference in swelling ratios between the reduced and oxidized states rely only on a change in hydrophilicity due to the charge number of the redox site without the help of an attractive intermolecular force by phase transition. However, it would be difficult to maintain a large difference in the swelling ratio between the reduced and oxidized states. Otherwise, it would be better to use a thermosensitive polymer with a higher LCST to maintain a large difference between the reduced and oxidized states by utilizing the phase transition at higher temperatures.

In order to induce self-oscillation while maintaining a larger amplitude at higher temperatures and around body temperature we prepared a self-oscillating gel composed of a thermosensitive $N, N$ '-ethylmethylacrylamide (EMAAm) polymer exhibiting a higher LCST than that of the NIPAAm polymer ${ }^{34}$. The self-oscillating behavior of the poly(EMAAm-co-Ru(bpy) $)_{3}$ ) gel was investigated by comparing against gels composed of a thermosensitive NIPAAm polymer with a lower LCST or non-thermosensitive $N, N^{\prime}$ dimethylacrylamide (DMAAm) polymer. It was shown that the poly(EMAAm-co-Ru(bpy) $)_{3}$ ) gel can induce swellingdeswelling self-oscillation while maintaining a larger ampli- tude near body temperature, while the other two gels do not undergo swelling-deswelling oscillation at that temperature (Fig. 8). The design concept of self-oscillation at higher temperatures without a decrease in swelling-deswelling amplitude was demonstrated by utilizing a thermosensitive polymer exhibiting a higher LCST.

\section{Concluding remarks}

As mentioned above, we proposed novel chemomechanical systems to convert chemical oscillation of the BZ reaction to mechanical changes of polymer and gel, and succeeded in realizing such an energy conversion system producing autonomous self-oscillation of polymer gel like a heart muscle. Here these recent progress on the self-oscillating polymers and gels and the design of functional material systems were summarized. As an innovative study to propose novel potential of polymer gels and achieve an autonomous behavior by coupling chemical and mechanical oscillations in polymer systems, the study has attracted much attention in the many research fields of polymer science, material science, physical chemistry, theoretical simulation, etc. as well as biophysics. We continue to advance our research by studying new polymer systems, which we hope to report in the future.

\section{Acknowledgements}

The author gratefully thanks all my students in the laboratory and the collaborators on this research project, especially to the present and previous Postdoc and Ph.D. students; Dr. Takeshi Ueki (Univ. of Tokyo), Dr. Daisuke Suzuki 
(Shinshu Univ.), Dr. Takamasa Sakai (Univ. of Tokyo), Dr. Yusuke Hara (AIST, Japan), Dr. Yasuhiro Maeda (Tokyo Medical and Dental Univ., Japan), Dr. Kosuke Okeyoshi (Univ. of Tokyo) and Dr. Yoko Murase (Dai Nippon Printing Co., Ltd., Japan), Dr. Tomonaga Ueno (Nagoya Univ., Japan).

\section{References}

1. Tanaka, T. Collapse of gels and the critical endpoint. Phys. Rev. Lett. 40, 820-823 (1978).

2. Yoshida, R. Design of functional polymer gels and their application to biomimetic materials. Curr. Org. Chem. 9, 16171641 (2005).

3. Ottenbrite, R. M., Park, K., Okano, T. \& Peppas, N. A. (Eds), Biomedical Applications of Hydrogels Handbook (Springer, New York, 2010).

4. Miyata, T. Stimuli-responsive polymer and gels. in Supramolecular Design for Biological Applications (Yui, N. ed.) pp. 191-225 (CRC Press, Boca Raton, 2002).

5. Lendlein, A. \& Shastri, V.P. Stimuli-sensitive polymers. $A d v$. Mater. 22, 3344-3347 (2010).

6. Field, R. J. \& Burger, M. (Eds.) Oscillations and Traveling Waves in Chemical Systems (John Wiley \& Sons, New York 1985).

7. Epstein, I. R. \& Pojman, J.A. An Introduction to Nonlinear Chemical Dynamics: Oscillations, Waves, Patterns, and Chaos (Oxford University Press, New York 1998).

8. Amemiya, T., Ohmori, T. \& Yamaguchi, T. An Oregonatorclass model for photoinduced behavior in the $\mathrm{Ru}(\mathrm{bpy})_{3}{ }^{2+}-$ catalyzed Belousov-Zhabotinsky reaction. J. Phys. Chem. A 104, 336-344 (2000).

9. Yoshida, R. Takahashi, T., Yamaguchi, T. \& Ichijo, H. Selfoscillating gel. J. Am. Chem. Soc. 118, 5134-5135 (1996).

10. Yoshida, R. Self-oscillating gels driven by the BelousovZhabotinsky reaction as novel smart materials. Adv. Mater. 22, 3463-3483 (2010).

11. Yoshida, R., Tanaka, M., Onodera, S., Yamaguchi, T. \& Kokufuta, E. In-phase synchronization of chemical and mechanical oscillations in self-oscillating gels. J. Phys. Chem. A 104, 7549-7555 (2000)

12. Maeda, S., Hara, Y., Yoshida, R. \& Hashimoto, S. Peristaltic motion of polymer gels. Angew. Chem. Int. Ed. 47, 66906693 (2008)

13. Shinohara, S., Seki, T., Sakai, T., Yoshida, R. \& Takeoka, Y. Chemical and optical control of peristaltic actuator based on selfoscillating porous gel. 39, Chem. Commun. 4735-4737 (2008).

14. Shinohara, S., Seki, T., Sakai, T., Yoshida, R. \& Takeoka, Y. Photoregulated wormlike motion of a gel. Angew. Chem. Int. Ed. 47, 9039-9043 (2008).

15. Tabata, O., Kojima, H., Kasatani, T., Isono, Y. \& Yoshida, R. Chemo-mechanical actuator using self-oscillating gel for artificial cilia. in Proceedings of the International Conference on MEMS 2003, pp. 12-15 (2003).

16. Maeda, S., Hara, T., Sakai, T., Yoshida, R. \& Hashimoto, S. Self-walking gel. Adv. Mater. 19, 3480-3484 (2007).

17. Kuksenok, O., Yashin, V. V., Kinoshita, M., Sakai, T., Yoshida, R. \& Balazs, A. C. Exploiting gradients in cross-link density to control the bending and self-propelled motion of active gels. J. Mater. Chem. 21, 8360-8371 (2011).

18. Yashin, V. V., Suzuki, S., Yoshida, R. \& Balazs, A.C. Controlling the dynamic behavior of heterogeneous self-oscillating gels. J. Mater. Sci. 22, 13625-13636 (2012).

19. Suzuki, D., Kobayashi, T., Yoshida, R. \& Hirai, T. Soft actu- ators of organized self-oscillating microgels. Soft Matter 8, 11447-11449 (2012).

20. Murase, Y., Maeda, S., Hashimoto, S. \& Yoshida, R. Design of a mass transport surface utilizing peristaltic motion of a self-oscillating gel. Langmuir 25, 483-489 (2009).

21. Murase, Y., Hidaka, M. \& Yoshida, R. Self-driven gel conveyer: Autonomous transportation by peristaltic motion of selfoscillating gel. Sensors and Actuators B 149, 272-283 (2010).

22. Murase, Y., Takeshima, R. \& Yoshida, R. Self-driven gel conveyer: effect of interactions between loaded cargo and selfoscillating gel surface. Macromol. Biosci. 11, 1713-1721 (2011).

23. Yoshida, R. \& Murase, Y. Self-oscillating surface of gel for autonomous mass transport. Colloids and Surfaces B: Biointerfaces 99, 60-66 (2012).

24. Shiraki, Y. \& Yoshida, R. Autonomous intestine-like motion of tubular self-oscillating gel. Angew. Chem. Int. Ed. 51, 6112-6116 (2012).

25. Yoshida, R. Sakai, T. Ito, S. \& Yamaguchi, T. Self-oscillation of polymer chains with rhythmical soluble-insoluble changes. J. Am. Chem. Soc. 124, 8095-8098 (2002).

26. Hara, Y. \& Yoshida, R. Self-oscillating polymer fueled by organic acid. J. Phys. Chem. B 112, 8427-8429 (2008).

27. Suzuki, D., Sakai, T. \& Yoshida, R. Self-flocculating/selfdispersing oscillation of microgels. Angew. Chem. Int. Ed. 47, 917-920 (2008).

28. Suzuki, D. \& Yoshida, R. Temporal control of self-oscillation for microgels by cross-linking network structure. Macromolecules 41, 5830-5838 (2008).

29. Suzuki, D. \& Yoshida, R. Self-oscillating core/shell microgels: effect of a crosslinked nanoshell on autonomous oscillation of the core. Polymer J. 42, 501-508 (2010).

30. Suzuki, D., Taniguchi, H. \& Yoshida, H. Autonomously oscillating viscosity in microgel dispersions. J. Am. Chem. Soc. 131, 12058-12059 (2009).

31. Taniguchi, H., Suzuki, D. \& Yoshida, R. Characterization of autonomously oscillating viscosity induced by swelling/deswelling oscillation of the microgels. J. Phys. Chem. B 114, 24052410 (2010).

32. Hara, Y. \& Yoshida, R. A viscosity self-oscillation of polymer solution induced by the BZ reaction under acid-free condition. J. Chem. Phys. 128, 224904 (2008).

33. Ueno, T., Bundo, K., Akagi, Y., Sakai, T. \& Yoshida, R. Autonomous viscosity oscillation by reversible complex formation of terpyridine-terminated poly(ethylene glycol) in the BZ reaction. Soft Matter 6, 6072-6074 (2010).

34. Hidaka, M. and Yoshida, R. Self-oscillating gel composed of thermosensitive polymer exhibiting higher LCST. J. Controlled Release 150, 171-176 (2011).

35. Dayal, R., Kuksenok, O. \& Balazs, A.C. Using light guide the self-sustained motion of active gels. Langmiur 25, 4298 4301 (2009)

36. Dayal, P., Kuksenok, O. \& Balazs, A.C. Designing autonomously motile gels that follow complex paths. Soft Matter $\mathbf{6}$, 768-773 (2010).

37. Yashin, V. V. Kuksenok, O. \& Balazs, A. C. Modeling autonomously oscillating chemo-responsive gels. Prog. Polym. Sci. 35, 155-173 (2010).

38. Yashin, V.V., Kuksenok, O., Dayal, P. \& Balazs, A.C. Mechano-chemical oscillations and waves in reactive gels. Rep. Prog. Phys. 75, 066601 (2012).

39. Lohmeijer, B. G. G. \& Schubert, U. S. Supramolecular engineering with macromolecules: An alternative concept for block copolymers. Angew. Chem. Int. Ed. 41, 3825-3829 (2002).

40. Yashin, V. V. Kuksenok, O. \& Balazs, A.C. Computational design of active, self-reinforcing gels. J. Phys. Chem. B 114, 6316-6322 (2010). 